\title{
Nonlinear dynamics and complexity in the generalized Lorenz system
}

\author{
Wiesław M. Macek $(\mathbb{D}$
}

Received: 2 October 2017 / Accepted: 22 August 2018 / Published online: 20 September 2018

(C) The Author(s) 2018

\begin{abstract}
Dynamics of magnetized fluids is much more complex than expected from the standard magnetohydrodynamics. Besides chaotic behavior that often appears in nonlinear dynamical systems, hyperchaotic motions are also possible on new strange attractors, with bifurcations which could result in turbulent irregular behavior. Surprisingly, all this complexity can be studied by analyzing a simple set of four ordinary differential equations describing hydromagnetic convection, which is the generalization of the famous Lorenz system for the case of turbulent convection in a fluid layer with the embedded magnetic field. This provides a novel contribution to chaos theory that could be of interest to the dynamical systems community.
\end{abstract}

Keywords Nonlinear dynamics - Hyperchaos · Strange attractors - Convection · Magnetohydrodynamics · Turbulence

\section{Introduction}

Dynamics of irregular flows in a viscous fluid belongs to the classical still unsolved open questions in math-

W. M. Macek $(\varangle)$

Faculty of Mathematics and Natural Sciences, Cardinal

Stefan Wyszyński University, Wóycickiego 1/3,

01-938 Warsaw, Poland

e-mail: macek@uksw.edu.pl

URL: www.cbk.waw.pl/ $\sim$ macek

W. M. Macek

Space Research Centre, Polish Academy of Sciences, Bartycka 18A, 00-716 Warsaw, Poland ematical physics, which notwithstanding of many efforts, including many numerical simulations, is as yet not sufficiently clear. Therefore, simplified models for some special cases, as for example for a fluid heated from below in a gravitational field with a vertical temperature gradient, are still very useful to grasp essential features of nonlinear dynamics. In this case of the Rayleigh-Bénard convection, the break through is attributed to Lorenz, who half a century ago, starting from complex basic hydrodynamic equations, obtained three simple but nonlinear ordinary differential equations [14]. As is well known, this famous paper has revealed complexity of nonperiodic deterministic flows, including strange attractors, chaotic behavior, bifurcations, and intermittency (see e.g., Ref. [27] for a review).

However, the standard Lorenz system does not take into account the influence of the anisotropy induced by the magnetic fields that can be embedded in some viscous flows appearing in nature. Several years ago, we have finally succeeded to generalize the Lorenz model for a horizontally magnetized fluid, with a new variable responsible for the induced magnetic field [16]. Admittedly, there is a huge difference between the horizontal and vertical directions of the magnetic fields and it seems that the later case is much more complicated to cope with and is still waiting for a reasonable approximated methods of solution. But in our next published letter we have shown that even in the case of horizontally magnetized layer the behavior of the solutions of our model could be quite complex and interest- 
ing [17]. Admittedly, to be applied in different regimes this model still requires much deeper analysis of various cases for broad range of parameters appearing in nature. Therefore, we hope that this new model could shed new light on chaos theory, nonlinear dynamics, and hopefully on hydromagnetic convection. In particular, the magnetohydrodynamical numerical codes used for real systems must reproduce the results of the generalized Lorenz model under the respective approximations.

Surprisingly, even such a deterministic simply magnetized system can exhibit rather complex behavior of solutions. It appears that by changing control parameters the system can easily go from equilibrium (described by a fixed point) or from periodic (limit cycles) to nonperiodic (chaotic) behavior; the influence of the applied magnetic field is not trivial. Naturally, besides the transitions induced by the changes in the parameters, all these types of behavior can be intertwined due to intermittent character of dynamics. Namely, within the theory of dynamical systems transitions from fixed points to periodic or nonperiodic flows often occur in a given system through bifurcations, intermittency, resulting in a turbulent irregular behavior of the nonlinear system. In fact, we have identified type I and III intermittency [20] in the generalized Lorenz model of hydromagnetic convection, as also discussed in the previous conference paper [15]. It would be interesting to look for the remaining basic type II intermittency and the respective Hopf bifurcation in this model.

Further, we know that two types of such nonperiodic flows are possible, namely chaotic and hyperchaotic motions. As first discovered by Lorenz in 1963, deterministic chaos exhibits sensitivity to initial conditions leading to unpredictability of the long-term behavior of the system, popularized as the so-called butterfly effect [14]. Hyperchaos is another type of a more complex nonperiodic flow, which has been for the first time identified in the generalized Lorenz model previously proposed by us in 2010 in Ref. [16]. More specifically, hyperchaos is defined as a complex nonperiodic behavior, where at least two Lyapunov exponents are positive in contrast to standard chaotic dynamics that is characterized by one positive Lyapunov exponent [22]. Admittedly, hyperchaotic behavior is only possible in at least four-dimensional systems, as discussed, e.g., in Ref. [12]. We have already identified such a behavior in our new model for hydromagnetic convection [17].
Please note that the aim of the previous work was simply the generalization of the Lorenz model and we have used the Prandtl number $\sigma$ equal to 10 (which is indeed standard for the classical Lorenz case), but is too high to be realistic. Therefore, in the present paper, we investigate the value of $\sigma=1$, which should be more appropriate for plasmas. Moreover, we discuss in detail mathematical and physical aspects of the derivation of the generalized Lorenz model and address thoroughly the physical character of underlying approximations. The results of this paper show how all these complex motions can be studied by analyzing our simple fourdimensional model. In particular, we discuss in detail irregular behavior of the system including new strange attractors, also in a hyperchaotic regime, and new types of bifurcations leading to turbulent irregular behavior of the nonlinear dynamical system.

\section{The convection equations}

It is known that viscous magnetized fluids should evolve according to three basic partial differential equations [11]:

$$
\frac{\mathrm{d} \mathbf{v}}{\mathrm{d} t}=-\frac{1}{\rho} \nabla\left(p+\frac{B^{2}}{2 \mu_{0}}\right)+\frac{(\mathbf{B} \cdot \nabla) \mathbf{B}}{\mu_{0} \rho}+v \nabla^{2} \mathbf{v}+\mathbf{f},
$$

$\frac{\mathrm{d} \mathbf{B}}{\mathrm{d} t}=(\mathbf{B} \cdot \nabla) \mathbf{v}+\eta \nabla^{2} \mathbf{B}$,

$\frac{\mathrm{d} T}{\mathrm{~d} t}=\kappa \nabla^{2} T$,

where $\nu, \eta$, and $\kappa$ denote, respectively, kinematic viscosity, magnetic diffusive viscosity (resistivity), and thermal conductivity of a given fluid. Besides the Navier-Stokes basic law, the second and third equation describe the magnetic advection-diffusion and the heat conduction, respectively. As usual for standard convection models, viscous diffusion is included in the term last but one in Eq. (1), magnetic resistivity in the last term in Eq. (2), and in Eq. (3) only thermal conductivity is retained, while the terms related to Ohmic and viscous heating are neglected, see, e.g., Ref. [3,26]. But even these somewhat simplified magnetohydrodynamical (MHD) equations are difficult to solve directly because both time and space changes, $\frac{\mathrm{d}}{\mathrm{d} t} \equiv \frac{\partial}{\partial t}+\mathbf{v} \cdot \nabla$, of the velocity $\mathbf{v}$ of the flow, the temperature $T$ together with mass density $\rho$ and pressure $p$, and the magnetic field $\mathbf{B}$ are considered. It is usually convenient to define 
Fig. 1 Schematic of geometry for hydromagnetic convection

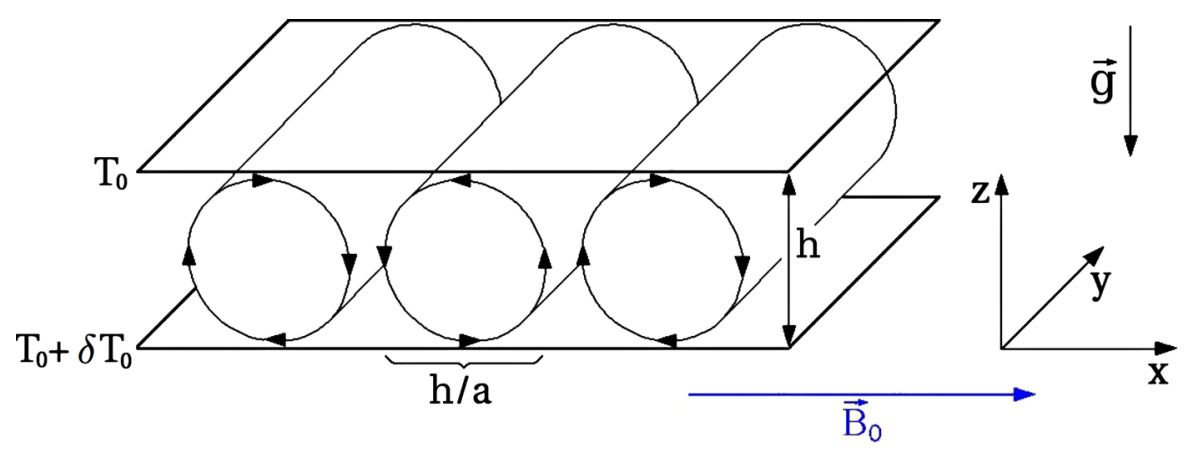

Alfvénic velocity $\mathbf{v}_{\mathrm{A}}=\mathbf{B} /\left(\mu_{0} \rho\right)^{1 / 2}$ with the constant magnetic permeability of free space $\mu_{0}$.

In Eq. (1) $\mathbf{f}$ denotes the volume density of additional external forces. In particular, for the Rayleigh-Bénard problem [21] under the vertical gravitational field with a constant acceleration $g$ this results in the buoyancy term $f=\rho g$. The schematics of this standard scenario of a horizontal ( $x$ axis) viscous fluid layer of height $h$ and aspect ratio $a$ is shown in Fig. 1 (no variations in $y$ direction), cf., e.g., Appendices to Refs. [3,26]. The fluid is heated from below with an applied vertical ( $z$ axis) temperature gradient, $\delta T_{0}$. As usual, taking a constant coefficient $\beta$, we take into account the volume expansion for $f$ term, $\rho=\rho_{0}\left[1-\beta\left(T-T_{0}\right)\right]$, but except that the fluid is treated as incompressible, $\rho=$ $\rho_{0}$ (the Oberbeck-Boussinesq approximation) $[4,19]$.

But now in comparison with classical RayleighBénard problem in our derivation, we take into consideration the effect of the magnetic field embedded in the fluid. Naturally, in the case of an incompressible fluid we can use a stream (potential) function $\Psi$ defined by $\mathbf{v}=\nabla \times \Psi$, and similarly a vector potential $\mathbf{A}$ for the magnetic field, $\mathbf{B}=\nabla \times \mathbf{A}$, satisfying naturally besides Eq. (2) the conditions $\nabla \cdot \mathbf{v}=0$ and $\nabla \cdot \mathbf{B}=0$.

One can expect that in the case of a thin horizontal layer, the influence of an external horizontal magnetic field should be important. If we apply an initial magnetic field $\mathbf{B}_{\mathbf{0}}$ (along the $x$ direction) by adding the Alfvén velocity $v_{\mathrm{A} 0}=B_{0} /\left(\mu_{0} \rho_{0}\right)^{1 / 2}$, while neglecting a possible vertical field, we can write the perturbed respective potentials in the forms: $\Psi=$ $\{0, \psi(x, z, t), 0\}$ and $\mathbf{A} /\left(\mu_{0} \rho_{0}\right)^{1 / 2}=\{0, \alpha(x, z, t)-$ $\left.v_{\mathrm{A} 0} z, 0\right\}$. As usual $\theta(x, z, t)$ describes a deviation from the linear temperature profile, $T(x, z, t)=T_{0}+$ $\delta T_{0}\left(1-\frac{h}{z}\right)+\theta(x, z, t)$ in Eq. (3).

In the case of two-dimensional flow, following the derivation by Saltzman [24], by taking rotation of Eq. (1), both the thermal and isotropic part of the magnetic pressure are obviously eliminated, but the anisotropic tension of the magnetic field lines $(\mathbf{B} \cdot \nabla) \mathbf{B} /\left(\mu_{0} \rho\right)$ should still be important. Next, it should be noted that the first term $(\mathbf{B} \cdot \nabla) \mathbf{v}$ on the right-hand side of Eq. (2) is responsible for changes of the flow velocity $\mathbf{v}$ in space at a given time along the convected magnetic field $\mathbf{B}$. This is apparently more important than a possible advection term $(\mathbf{v} \cdot \nabla) \mathbf{B}$, as justified, e.g., in Ref. [6] (for a constant magnetic field $\mathbf{B}_{\mathbf{0}}$ this term vanishes). Therefore, for the case of the convective movement of the magnetic field frozen-in a fluid only the convection term $(\mathbf{B} \cdot \nabla) \mathbf{v}$ is used here, as argued in Ref. [16]. In this way, using curl operator of the basic equations (1)-(3) one obtains the following equations for the stream potential $\psi$, the magnetic perturbed potential $\alpha$, and the departure from linear temperature profile $\theta$ (i.e., with no convection rolls):

$$
\begin{aligned}
\frac{\partial}{\partial t} \nabla^{2} \psi= & -\frac{\partial\left(\psi, \nabla^{2} \psi\right)}{\partial(x, z)}+\frac{1}{\mu_{0} \rho} \frac{\partial\left(\alpha, \nabla^{2} \alpha\right)}{\partial(x, z)} \\
& +\frac{B_{0}}{\mu_{0} \rho} \frac{\partial}{\partial x} \nabla^{2} \alpha \\
& +v \nabla^{4} \psi+g \beta \frac{\partial \theta}{\partial x} \\
\frac{\partial}{\partial t} \nabla^{2} \alpha= & -\frac{\partial\left(\psi, \nabla^{2} \alpha\right)}{\partial(x, z)}+\frac{\partial\left(\alpha, \nabla^{2} \psi\right)}{\partial(x, z)}+B_{0} \frac{\partial}{\partial x} \nabla^{2} \psi \\
& +\eta \nabla^{4} \alpha+2 \frac{\partial^{2} \psi}{\partial x \partial z}\left(\frac{\partial^{2} \alpha}{\partial x^{2}}-\frac{\partial^{2} \alpha}{\partial z^{2}}\right) \\
& -2 \frac{\partial^{2} \alpha}{\partial x \partial z}\left(\frac{\partial^{2} \psi}{\partial x^{2}}-\frac{\partial^{2} \psi}{\partial z^{2}}\right)
\end{aligned}
$$

and

$$
\frac{\partial}{\partial t} \theta=-\frac{\partial(\psi, \theta)}{\partial(x, z)}+\frac{\delta T_{0}}{h} \frac{\partial \psi}{\partial x}+\kappa \nabla^{2} \theta
$$


where the Jacobian operator is defined by

$$
\frac{\partial(a, b)}{\partial(x, z)} \equiv \frac{\partial a}{\partial x} \frac{\partial b}{\partial z}-\frac{\partial a}{\partial z} \frac{\partial b}{\partial x},
$$

and the fourth-order operator is given by

$$
\nabla^{4} \equiv\left(\nabla^{2}\right)^{2}=\frac{\partial^{4}}{\partial x^{4}}+\frac{\partial^{4}}{\partial z^{4}}+2 \frac{\partial^{4}}{\partial x^{2} \partial z^{2}}
$$

(note an error in the formula given in Appendix of Ref. [26]). Third and fourth terms on the right-hand side of Eq. (4) result from the second term of Eq. (1), Eq. (5) is new, and Eq. (6) is the same as for unmagnetized fluid, see Ref. [14]. We use free boundary conditions, similarly as for the standard classical Lorenz model, i.e., all three functions $\psi, \alpha$, and $\theta$, and their Laplacian operators should vanish at both $z=0$ and $z=h$.

\section{The generalized Lorenz model}

Because both the velocity and the magnetic field in Eqs. (1) and (2) satisfy the same conditions $\nabla \cdot \mathbf{v}=0$ and $\nabla \cdot \mathbf{B}=0$, the Fourier representations should be similar for both velocity and magnetic potentials. Therefore, following Rayleigh [21] we can look for solutions of the potentials $\psi$ and $\alpha$ for both the bulk and the Alfvén velocities, in the double asymmetric (taken into account by parameter $a$ ) Fourier representation [24]

$$
\begin{aligned}
& \psi(x, z, t)=\frac{1+a^{2}}{a} \kappa \sqrt{2} X(t) \sin \left(\frac{\pi a}{h} x\right) \sin \left(\frac{\pi}{h} z\right), \\
& \alpha(x, z, t)=\frac{1+a^{2}}{a} \kappa \sqrt{2} W(t) \cos \left(\frac{\pi a}{h} x\right) \sin \left(\frac{\pi}{h} z\right) .
\end{aligned}
$$

Certainly, as seen in Eq. (3) the temperature is of somewhat different character, and for the corresponding function $\theta$ we retain the same first and second order terms as originally used by Lorenz [14].

$$
\begin{aligned}
\theta(x, z, t)= & \frac{R_{c}}{\pi R_{\mathrm{a}}} \delta T_{0} \sqrt{2} \\
& \left(Y(t) \cos \left(\frac{\pi a}{h} x\right) \sin \left(\frac{\pi}{h} z\right)\right. \\
& \left.-Z(t) \sin \left(\frac{2 \pi}{h} z\right)\right)
\end{aligned}
$$

In the well-known three-dimensional Lorenz model, besides a time-dependent variable $X$ proportional to the intensity of the convective motion, the other two variables $Y$ and $Z$ describe the temperature profile in Eq. (3), see Ref. [14]. In addition, in the case of the magnetized fluid we have introduced a new time-dependent variable $W$ describing the profile of the magnetic field induced in the convected fluid according to Eqs. (1) and (2) as described in Eq. (10).

Using the approximation $(\mathbf{B} \cdot \nabla) \mathbf{v} \approx\left(\mathbf{B}_{\mathbf{0}} \cdot \nabla\right) \mathbf{v}$ in Eq. (2), we have obtained from the general hydromagnetic Eqs. (1)-(3) a model described by four ordinary differential equations [16]:

$$
\begin{aligned}
\dot{X} & =-\sigma X+\sigma Y-\omega_{0} W, \\
\dot{Y} & =-X Z+r X-Y, \\
\dot{Z} & =X Y-b Z, \\
\dot{W} & =\omega_{0} X-\sigma_{\mathrm{m}} W,
\end{aligned}
$$

where dots denote derivatives with respect to the normalized time $t^{\prime}=\left(1+a^{2}\right) \kappa(\pi / h)^{2} t$, while $\sigma=v / \kappa$ is the Prandtl number (ratio of the kinematic viscosity to the thermal conductivity), and $b=4 /\left(1+a^{2}\right)$. As usual $r=R_{\mathrm{a}} / R_{\mathrm{c}}$ is a control parameter of the system proportional to the temperature gradient $\delta T_{0}$, or a Rayleigh number $R_{\mathrm{a}}=g \beta h^{3} \delta T_{0} /(v \kappa)$ normalized by a critical number $R_{\mathrm{c}}=\left(1+a^{2}\right)^{3}\left(\pi^{2} / a\right)^{2}$.

In addition to the standard Lorenz system [14], we have introduced another control parameter proportional to the initial magnetic field strength $B_{0}=\left|\mathbf{B}_{\mathbf{0}}\right|$ applied to the system, along the $\mathrm{x}$ axis in Fig. 1, that is defined here as a basic dimensionless magnetic frequency $\omega_{0}=v_{\mathrm{A} 0} / v_{0}$, with $v_{0}=4 \pi \kappa /(a b h)$. The last term in Eq. (12) comes from the anisotropic tension of the magnetic field lines $(\mathbf{B} \cdot \nabla) \mathbf{B} /\left(\mu_{0} \rho\right)$ in Eq. (1). Similarly, the first term of Eq. (15) results from $(\mathbf{B} \cdot \nabla) \mathbf{v}$ on the right-hand side of Eq. (2), describing the flow changes along the convected magnetic fields, see Ref. [16]. Naturally, besides the Prandtl number $\sigma=v / \kappa$, the properties of the magnetized fluid are characterized by an analogue parameter of resistive viscosity $\sigma_{\mathrm{m}}=\eta / \kappa$ (ratio of resistivity and conductivity) appearing now in Eq. (15), and resulting from the last terms in Eqs. (2) and (3). It is worth noting that coupling the first and fourth equations of the generalized Lorenz system, Eqs. (12) and (15), and the second and third Eqs. (13) and (14), gives two pairs looking very similar and are mutually interconnected by single variables $(\mathrm{W}, \mathrm{X})$ and (Z, Y). Therefore, the four-dimensional system seems to be even more symmetric than the classical threedimensional Lorenz model. 
Admittedly, we have verified that in the case for the inclusion of any higher order terms, one would need to consider a wider spectrum of modes certainly not limited to Eqs. (9-11). Following a basic derivation by Saltzman [24], a number of truncated $n$ th-order models of convection has been studied. However, one should bear in mind fundamental difficulties in detecting lowdimensional behavior in experimental/observational data for the correlation dimension greater than approximately five [23]. For dynamical systems of dimensionality above this threshold, time series analysis methods do not allow to distinguish properly between lowdimensional dynamics and random data, especially in the presence of observational dynamical noise [28,29]. Therefore one can expect fundamental problems with falsifiability of five-and-more dimensional models. In this context, the low-dimensional model proposed in our paper provides an interesting falsifiable alternative exhibiting surprisingly rich dynamical behavior already in the four-dimensional phase space [16].

\section{Analysis of the model}

By using the generalized Lorenz system we can write Eqs. (12) and (15) as given in Ref. [16]

$$
\begin{aligned}
& \ddot{X}+\sigma \dot{X}+\left(\sigma r-\omega_{0}^{2}\right) X=-\sigma(Y+X Z)+\sigma_{\mathrm{m}} \omega_{0} W, \\
& \ddot{W}+\sigma_{\mathrm{m}} \dot{W}+\omega_{0}^{2} W=\sigma \omega_{0}(Y-X) .
\end{aligned}
$$

We see that both variables $X$ and $W$ satisfy the equations of two coupled damped linear oscillators. However, the terms on the right-hand side of Eqs. (16) and (17) may be interpreted as nonlinear driving forces. But now the coupling between $X, W$ and $Y, Z$ is enhanced owing to the magnetic field $\mathbf{B}$. In particular, when $\omega_{0}=0$ this coupling ceases and the variable $W$ is damped by the magnetic viscosity, see Eqs. (12) and (15).

\subsection{Stability of the fixed points and bifurcations}

The fixed points of the generalized Lorenz system, Eqs. (12)-(15), can be obtained similarly as for the standard Lorenz system reported in Appendix B in Ref. [26]. As usual we start with a trivial zero fixed point $C^{0}=(0,0,0,0)$ and two nonzero fixed points $C^{ \pm}$. Now, defining the quantity $e=\omega_{0}^{2} /\left(\sigma \sigma_{\mathrm{m}}\right)$ for the magnetic control parameter $\omega_{0}^{2}$, and the values characterizing the usual kinetic and magnetic properties of the fluid $\sigma$ and $\sigma_{\mathrm{m}}$, we see that the fixed point $C^{0}$ is stable for $0 \leq r<r_{0}$, where the critical value $r_{0}=1+e$ can be interpreted as a characteristic value of the Rayleigh number for the onset of convection at the pitchfork bifurcation. Besides that there is another value of $r_{\mathrm{T}}$, so-called the Takens-Bogdanov point [30], satisfying the condition

$\omega_{0}^{2}=\sigma_{\mathrm{m}}^{2}(1+\sigma) /\left(1-\sigma_{\mathrm{m}}\right) r_{\mathrm{T}}=\left(\sigma+\sigma_{\mathrm{m}}\right) / \sigma /\left(1-\sigma_{\mathrm{m}}\right)$

at which, if $\omega_{0}^{2}$ is greater than the value given in Eq. (18), the fixed point $C^{0}$ undergoes a Hopf bifurcation, that can be responsible for chaotic solutions.

On the other hand, the nonzero fixed points are given by

$$
\begin{aligned}
C^{ \pm}= & \pm d / \sqrt{1+e}, \pm d \sqrt{(1+e)}, r-(1+e), \\
& \left. \pm\left(\sigma / \omega_{0}\right) d e / \sqrt{1+e}\right\},
\end{aligned}
$$

with $d=\sqrt{b[(r-1)-e]}$ [16]. The additional fixed points $C^{ \pm}$are stable for $r_{0} \leq r<r_{H}$, where $r=r_{H}$ is a critical value at which the system looses stability and a Hopf bifurcation takes place. Naturally, the critical number $r_{0}$ for the onset of convection increases with the applied magnetic field $B_{0}$, thus the magnetic field can stabilize the convection as regards to the appearance of convective rolls. However, if we consider oscillations of the convection rolls as described by the model of Eqs. (12)-(15), the influence of the induced magnetic field on the fluid motion is much more complex than only suppressing of dynamics as expected from the standard textbooks of magnetohydrodynamics, cf., e.g., $[6,11]$.

Also Hopf bifurcation of trivial equilibrium is interesting compared to classical case. Admittedly, stability of the generalized four-dimensional Lorenz system can be analyzed by looking for roots of the characteristic equation, which is a fourth-order polynomial

$P(\lambda)=\lambda^{4}+a_{3} \lambda^{3}+a_{2} \lambda^{2}+a_{1} \lambda+a_{0}=0$

with the following coefficients depending on the parameters of the model: $a_{0}=2 b\left((r-1) \sigma_{\mathrm{m}} \sigma-\omega_{0}^{2}\right)$, $a_{1}=b\left(\sigma(r-1+c)+\sigma_{\mathrm{m}}(\sigma+1+c)+\omega_{0}^{2}\left(1-2 / \sigma_{\mathrm{m}}\right)\right)$, $a_{2}=b\left(\sigma_{\mathrm{m}}+\sigma+c+1\right)+\sigma_{\mathrm{m}}(\sigma+1)+\omega_{0}^{2}\left(1-1 / \sigma_{\mathrm{m}}\right)$, and $a_{3}=\sigma_{\mathrm{m}}+\sigma+b+1$, where $c=\left(\sigma_{\mathrm{m}} \sigma(r-1)-\right.$ 
$\left.\omega_{0}^{2}\right) /\left(\sigma_{\mathrm{m}} \sigma+\omega_{0}^{2}\right)=r / r_{0}-1$. The system is stable as long as the real parts of all the eigenvalues $\left(\lambda_{i}\right.$, $i=1, \ldots, 4)$ are smaller than zero.

In principle, even in this more complicated case the solution for the Hopf bifurcation $r_{H}$, where the polynomial has a pair of pure imaginary eigenvalues $\left(\lambda_{1,2}= \pm i \omega\right)$, can be obtained analytically. However, for any given control parameter $\omega_{0}$, the obtained general form for $r_{H}$ appears to be a very long and tedious expression depending on the other three parameters of the model, i.e., the magnetic Prandtl number $\sigma_{\mathrm{m}}$, the Prandtl number $\sigma$, and the geometric parameter $b$. Only given fixed standard values (usually $\sigma=10$ and $b=$ $8 / 3$ are used) this formula for $r_{H}$ as a function of $\sigma_{\mathrm{m}}$ can somewhat be simplified to several lines. Obviously, one can immediately notice that for unmagnetized fluid (with $\omega_{0}=0$ and $\sigma_{\mathrm{m}}=0$ ) we have $c=r-1$ and $a_{0}=0$, and hence Eq. (20) is reduced to the third-order polynomial (with $a_{1}=2 b \sigma(r-1), a_{2}=b(\sigma+r)$, and $\left.a_{3}=\sigma+b+1\right)$. In this way we can verify that the wellknown simple formula $r_{H}=\sigma(\sigma+b+3) /(\sigma-b-1)$ for the standard three-dimensional Lorenz system can be recovered, with a value of $r_{H}=24.74$ for $\sigma=10, b$ $=8 / 3$, Ref. [26]. When the control parameter $r$ exceeds this value, the real parts of the eigenvalues are positive and the fixed points looses stability, resulting in a chaotic behavior of the solutions of the classical Lorenz system.

On the other hand, in the generalized Lorenz system this direct transition to chaos is possible only for a certain range of the embedded magnetic field described by the magnetic control parameter $\omega_{0}$, which includes also the particle density of the fluid. Namely, as is illustrated, e.g., in Fig. 1 of [16], for a given magnetic Prandtl number, $\sigma_{\mathrm{m}}=1$, three different regions of long-term dynamical behaviors are possible, namely fixed (equilibrium) points, periodic (limit cycles), and nonperiodic (chaotic) motions. The Hopf bifurcations are expected at the horizontal line limiting the fixed point solutions; the vertical line separates periodic from nonperiodic (chaotic) solutions.

We see that for small $\omega_{0}(\lesssim 4)$ we can have a direct transition from a fixed point to chaotic dynamics, but for somewhat higher magnetic field, e.g., for $\omega_{0}=5$, our model predicts a transition to periodic behavior at some value of the Rayleigh number (related with the temperature gradient included in the control parameter $r$ ), and next a transition from periodic to chaotic behavior for some larger value of $r=r_{H}$. It is somewhat surprising that for a fixed temperature gradient (e.g., for $r=20$ ), by increasing the strength of the embedded magnetic field one can induce a chaotic behavior, but the stronger magnetic fields can damp again this behavior, according to Eq. (16), when the last term in the left-hand side changes sign. We can hence expect that various types of the Hopf bifurcations are possible in the generalized Lorenz system [16]. Obviously, when the fixed points loose stability, the bifurcation diagrams becomes extremely complex with intertwined chaotic and various periodic regimes, including period three windows, which as is known could imply chaos [13,25]. This is, however, beyond the scope of this basic paper, and should be subject of another detailed studies in the near future.

\subsection{Long-term behavior}

In Refs. $[16,17]$ the behavior of the dynamical system of Eqs. (12)-(15) was discussed in detail in the space of dimensionless control parameters $\omega_{0}$ and $r$ for the Prandtl number $\sigma=10$. The analysis revealed a variety of possible dynamical behavior including chaos, hyperchaos and intermittency. References $[16,17]$ are aimed at possibly direct comparison of dynamical properties of the hydromagnetic Lorenz model with the standard Lorenz equations. Therefore, the Prandtl number was assumed $\sigma=10$, which is a common value used in the literature for the standard Lorenz model. However for real plasmas $\sigma \approx 1$ is a more realistic assumption $[7,9,10]$. For $\sigma=v / \kappa \approx 1$ the kinematic viscosity $v$ and thermal diffusivity $\kappa$ are approximately equal, which implies comparable diffusion rates of the heat and the momentum. Physically this new case is significantly different from previously analyzed case $(\sigma=10)$, where the diffusive transfer of momentum was dominant as compared to the heat transfer.

In Fig. 2 we present plots of the largest Lyapunov exponent in the space of dimensionless control parameters $\omega_{0}$ and $r$ for $\sigma=1$. The plots illustrate longterm (asymptotic) behavior of the dynamical system of Eqs. (12)-(15) in the parameter space. The Lyapunov exponents are computed for solutions of Eqs. (12)-(15) using the QR decomposition method (discussed thoroughly in Sec. VC of Ref. [8]) that provides reliable and accurate estimation of the full spectrum of the exponents when differential equations are explicitly known. The method requires long time series (that naturally 

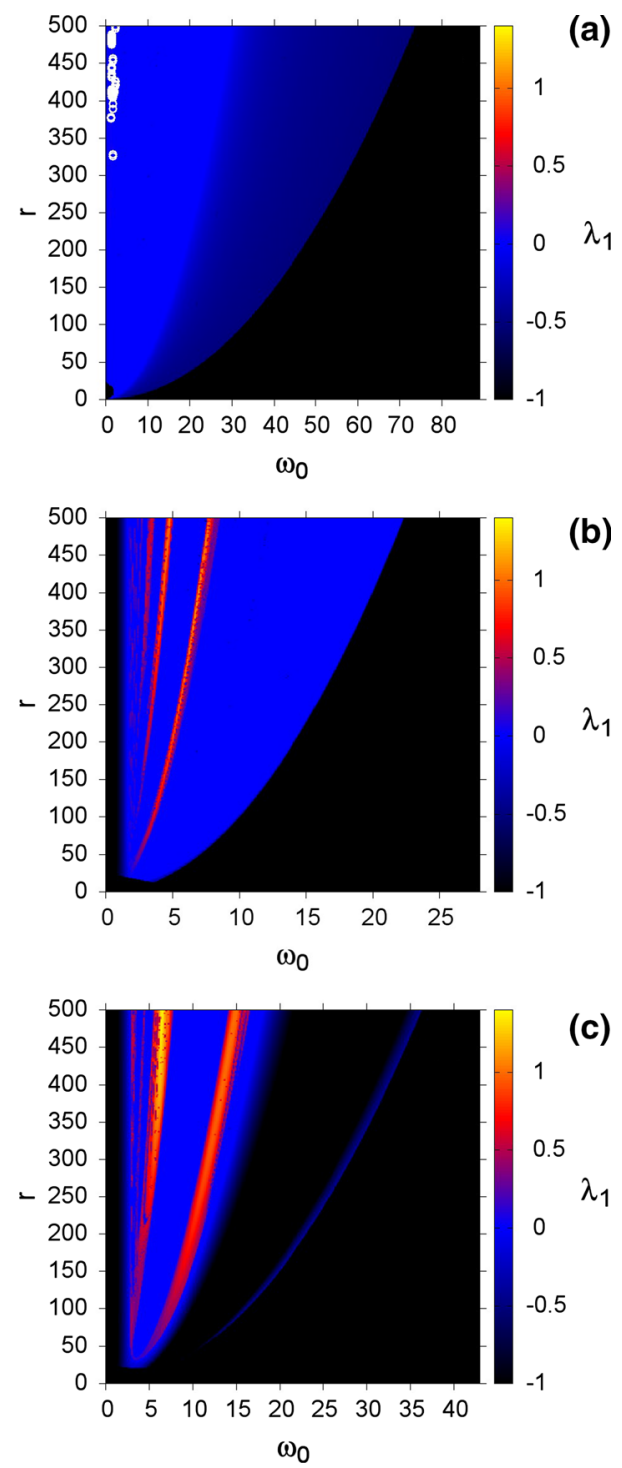

Fig. 2 Dependence of the largest Lyapunov exponent $\lambda_{1}$ (colorcoded) on $\omega_{0}$ and $r$ parameters of the generalized Lorenz model for $\mathbf{a} \sigma_{\mathrm{m}}=0.1, \mathbf{b} \sigma_{\mathrm{m}}=1$, and $\mathbf{c} \sigma_{\mathrm{m}}=3$. Other parameters of the system have fixed values: $\sigma=1, b=8 / 3$. Regions of convergence of the solutions of Eqs. (12)-(15) to fixed points $\left(\lambda_{1}<0\right)$ are shown in color scale from black to blue, to periodic solutions $\left(\lambda_{1}=0\right)$ - in blue, to chaotic solutions $\left(\lambda_{1}>0\right)$-in a color, consistently with the color bar scale, from blue to yellow. White circles for $r>300$ and $\omega_{0} \approx 2$ in panel (a) show weakly chaotic solutions $\left(\lambda_{1}>0.1\right)$ for $\sigma_{\mathrm{m}}=0.1$

appear in the case of periodic or chaotic solutions); thus a preparatory step has been applied to detect convergence of a given solution to a fixed point. Depending on the sign and value of the calculated largest Lyapunov exponent, various regions of convergence are shown in different colors. Namely, fixed points (lack of longterm limit cycle oscillations, negative exponent) are indicated in color scale from black to blue, periodic solutions (zero value) - in blue, and chaotic dynamics (with positive exponent)—in a color, consistently with the color bar scale, from blue to yellow, respectively. Three cases of the parameter $\sigma_{\mathrm{m}}=\eta / \kappa=\sigma / P r_{\mathrm{m}}$ (where $P r_{\mathrm{m}}=v / \eta$ is the magnetic Prandtl number) are considered here as related to different magnitude of resistive dissipation affecting the system. We are aware that the range of these values is rather broad both for laboratory and space plasmas. Therefore, the results obtained for chosen values from 0.1 to 3 are only shown here for illustration.

For $\sigma_{\mathrm{m}}=0.1$ (Fig. 2a) the most part of the plot is black or dark blue, which means that the solutions converge to fixed points corresponding to equilibria, and long-term limit cycle oscillations are not possible. The region of periodic (limit cycle) and nonperiodic (chaotic) solutions seen in light blue is located mostly in the left top part of the plot. Weakly chaotic solutions $\left(\lambda_{1}>0.1\right.$, marked by white circles) are found for $r>300$ and $\omega_{0} \approx 2$. Note that when we move from weakly chaotic or periodic dynamics for small values of $\omega_{0}$ (light blue region) to equilibria region (dark blue and black), the transition is smooth for increasing $\omega_{0}$. For $\sigma_{\mathrm{m}}=1$ (Fig. 2b) the structure of the plot is significantly different. Solutions converge to equilibria for both small and large values of $\omega_{0}$. When we move to the region of periodic solutions starting from large values of $\omega_{0}$, the transition between equilibria black region and periodic solutions is sharp. On the other hand, when we move from equilibria to periodic solutions starting from small values of $\omega_{0}$, the transition is smooth. In the region of periodic solutions we can see stripes of chaotic behavior (a color from light blue to yellow, consistently with the color scale). More detailed inspection of the plots reveals complicated structure of the stripes, where chaotic solutions are intertwined finely with domains of periodic solutions. For $\sigma_{\mathrm{m}}=3$ (Fig. 2c) the plot is generally similar to Fig. 2b. However, when we move to the region of periodic solutions starting from large values of $\omega_{0}$, the transition between equilibria (black region) and periodic solutions is smooth for Fig. 2c, whereas sharp change is seen for Fig. $2 b$ as discussed above. Another difference concerns the presence of a blue strip along the diagonal of Fig. 2c, which is not seen in Fig. 2b. Note that the fine structure in the chaotic region of the parameter 
space seen for $\sigma_{\mathrm{m}} \geq 1$ implies interesting properties of the dynamics as regards to regularity of convective motions, when affected by changing boundary conditions related to control parameters $r$ and $\omega_{0}$ used in the model.

The influence of the external magnetic field $B_{0}$ on the dynamics in the model can be studied for fixed value of $r$ by changing the control parameter $\omega_{0}$, which is related to $B_{0}$. As seen in Fig. 2a for small magnetic diffusivity we generally observe a transition from chaotic (or periodic) dynamics to fixed point solutions for the increasing $\omega_{0}$ parameter. This suggests purely damping influence of the increasing external magnetic field. For larger magnetic diffusivity (Fig. 2b and c) we observe a more complicated scenario of transitions. For small $\omega_{0}$ the solutions converge to fixed points, for larger values of $\omega_{0}$ we can see intertwined periodic and chaotic regions. For even larger values of $\omega_{0}$ the transition is seen from periodic dynamics to fixed points convergence. Except for this general dynamical pattern in the control parameters $\omega_{0}$ and $r$ space, we can see a fine structure of the chaotic regions. The external temperature gradient $\delta T_{0}$ also influences the dynamics in an intricate manner for some range of values of $\omega_{0}$ as seen in Fig. 2 analyzing the dependence of solutions on $r \propto \delta T_{0}$ for fixed values of $\omega_{0}$.

Similar analysis as presented above was done for $\sigma=10$ in Ref. [17], which allows us to compare the case for large diffusive momentum transfer $(\sigma=10)$ with the case of comparable diffusion rates of the heat and the momentum $(\sigma=1)$. The following differences are seen when we compare the two cases. For $\sigma=10$ and $\omega_{0} \approx 0$ periodic or chaotic dynamics is observed in wide range of $r$ parameter values [17]. This kind of behavior is seen for $\sigma=1$ but only for small magnetic diffusivity implying $\sigma_{\mathrm{m}}=0.1$ (Fig. 2a), whereas for $\sigma_{\mathrm{m}}=1$ and $\sigma_{\mathrm{m}}=3$ (Fig. $2 \mathrm{~b}$ and c) solutions converge to fixed points for $\omega_{0} \approx 0$. Moreover for $\sigma=10$ the region of periodic or chaotic solutions extends for wider range of $\omega_{0}$ in comparison with $\sigma=1$ case. The structure of chaotic regions is more striped in the case of $\sigma=1$ than for $\sigma=10$. Another difference concerns transitions in the control parameters $\omega_{0}$ and $r$ space between regions of periodic solutions and convergence to fixed points. For $\sigma=10$ the transitions are sharp [17], whereas more gradual changes are observed for $\sigma=1$ as discussed in this paper.

\subsection{Chaotic strange attractors}

Integrating Eqs. (12)-(15) of the system for hydromagnetic convection and projecting the four-dimensional solutions onto the three-dimensional subspace spanned by $X, Y$, and $W$ axes, we can get some imagination about the complex structure of dynamically interesting strange attractors of the system. Some of the typical calculated shapes are presented in Fig. 3. Naturally, the obtained set should depend on the values of the assumed five parameters. For simplicity, we take here the standard values of the classical Lorenz model parameters: $r=28, \sigma=10$, and $b=8 / 3$. Now, with a magnetic field, $\omega_{0}=1$, and a small magnetic viscosity, $\sigma_{\mathrm{m}} \approx 0$, we can verify that the obtained butterflyshaped structure strongly wanders along the $W$ axis, case (b), as taken from [16, Fig. 2a]. As is known that for dissipative systems the volume in phase space shrinks rapidly due to both kinematic and magnetic viscosities, $\dot{V}=-\left(\sigma+\sigma_{\mathrm{m}}+b+1\right) V$; the attractor is strange because it has a measure zero. Therefore, as Lorenz underlines because of dissipation the trajectories only appears to merge, but they actually remain distinct [14]. Here the shrinking of volume is only enhanced owing to magnetic viscosity $\sigma_{\mathrm{m}}$.

We recall a structure for $\omega_{o}=6$ in the presence of some magnetic viscosity, $\sigma_{\mathrm{m}}=2$, which is presented in Fig. 3c taken from [16, Fig. 2b]. It is interesting to note that this strange attractor appears when changing the magnetic control parameter in some narrow range near $\omega_{0}=6$. We see that trajectories in the phase space describing the perturbed magnetic vector potential merge and separate again resulting in irregularly reappearing 'islands'. This merging is related to a special (hyper)-surface, which separates small oscillations around one of two fixed points, $C^{ \pm}$, from large oscillations that encircle all fixed points, including a zero unstable fixed point, $C^{0}$. Therefore, in the vicinity of this value a periodic motion is interrupted with chaotic bursts, as shown in Fig. 2 of Ref. [15]. If the magnetic field strength is further increased so that the last term in the left-hand side of Eq. (16) changes sign, $\omega_{0}^{2}>\sigma r$, the oscillations are depressed and the system will tend to a fixed point as shown in Fig. 1 of Ref. [16]. Here another new interesting strange attractor for $\omega_{0}=5$, $\sigma_{\mathrm{m}} \approx 0$ is also depicted in Fig. $3(\mathrm{~d})$. 
(a)

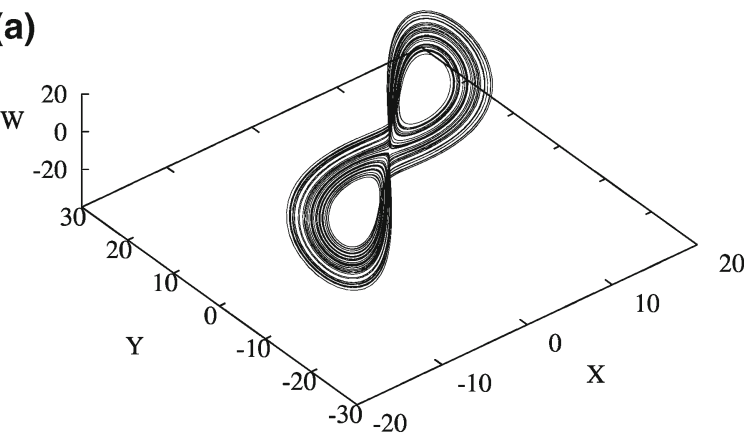

(c)

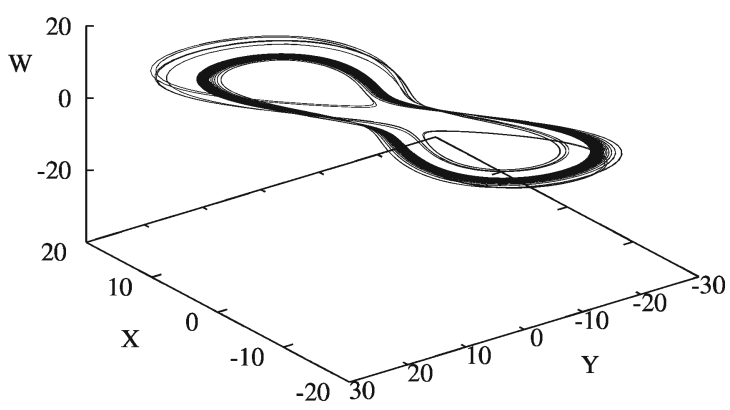

(b)

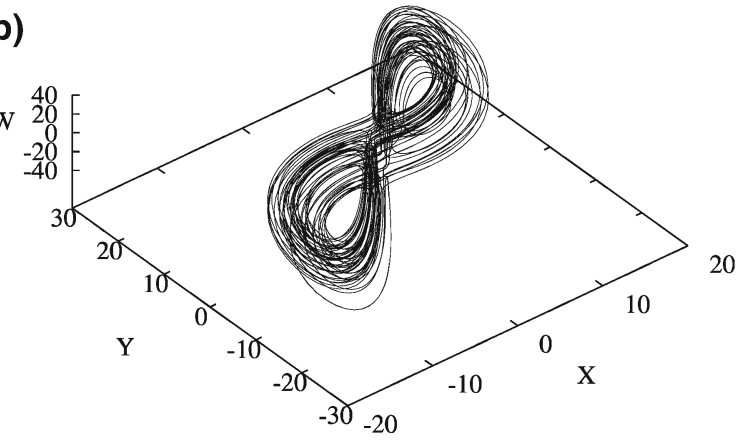

(d)

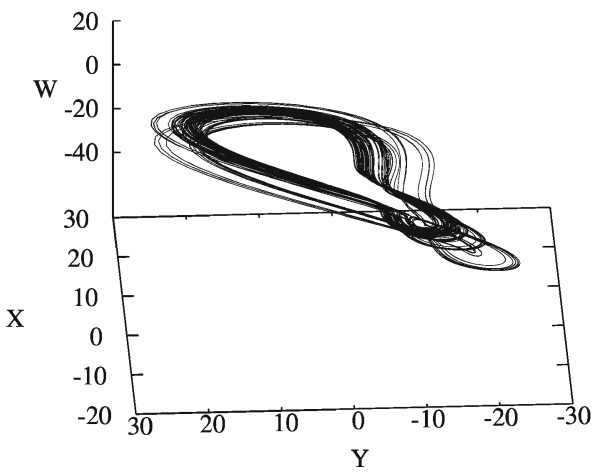

Fig. 3 The three-dimensional projection of the attractor for $\mathbf{a} \omega_{0}=1, \sigma_{\mathrm{m}}=20, \mathbf{b} \omega_{0}=1, \sigma_{\mathrm{m}} \approx 0, \mathbf{c} \omega_{0}=6, \sigma_{\mathrm{m}}=2$, and $\mathbf{d} \omega_{0}=5$, $\sigma_{\mathrm{m}} \approx 0$, respectively

\subsection{Hyperchaotic strange attractors}

According to the Liouville theorem the sum of all Lyapunov exponents cannot be positive (for any continuous system one of the local exponents is zero), hence two positive Lyapunov exponents can only appear for at least four-dimensional systems. In this case the dynamics is called hyperchaotic. It is obvious that this is not possible for the classical standard three-dimensional Lorenz system.

However, in the four-dimensional generalized Lorenz system for convection in the magnetized fluid, hyperchaotic solution is, in principle, possible owing to a new variable describing the induced magnetic field. In fact, we have identified hyperchaos only for some limited range of values of the external temperature gradient $r$ and the applied magnetic field (with parameter $\omega_{0}=5.95$ ), for fixed other parameters $\sigma=10$, $\sigma_{\mathrm{m}}=0.1, b=8 / 3$, see Fig. 2 of Ref. [17]. Namely, it appears that the largest Lyapunov increases abruptly becoming positive for $r \geq 454.7$ (except of a gap for $461.8<r<462.4$ with periodic solutions), while at the same onset value the second Lyapunov exponent becomes positive, increasing smoothly.

This exhibits a clear transition to hyperchaotic dynamics. One can expect that in the generalized Lorenz system this transition results from nonlinear coupling between the anisotropic tension of the magnetic field lines and the magnetic viscosity. In this paper in Fig. 4 we show the three-dimensional projections of the hyperchaotic attractor to four subspaces of the phase space. The parameter values for the hyperchaotic attractor are taken accordingly to the values reported in Ref. [17]: $r=480, \omega_{0}=5.95, \sigma=10, \sigma_{\mathrm{m}}=0.1$, and $b=8 / 3$.

These results may request an experimental identification of hyperchaotic dynamics in laboratory and also space plasmas, including magnetoconfined plasmas in nuclear fusion devices or tokamaks [1], possibly in nanotechnology, and also solar sunspots [18] and planetary or stellar fluid interiors [5]. However, it could rather be difficult to identify such a system in nature because of some specific experimental problems. Namely, at least some trajectories of any hyperchaotic system must diverge in two different direc- 

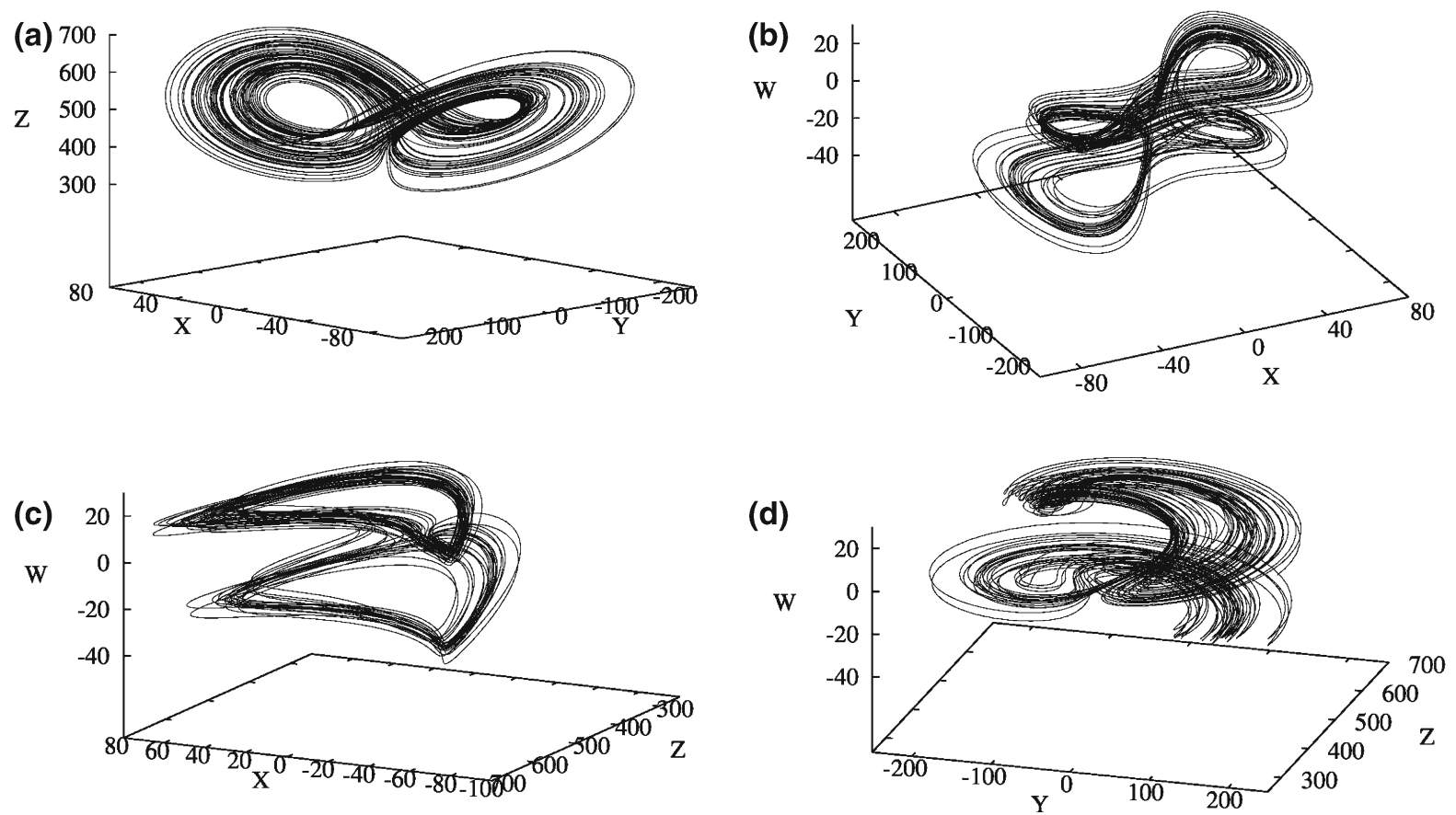

Fig. 4 The three-dimensional projections of the hyperchaotic attractor to the subspaces a XYZ, b XYW, $\mathbf{c} X Z$, and d YZW for $r=480, \omega_{0}=5.95, \sigma=10, \sigma_{\mathrm{m}}=0.1$, and $b=8 / 3$

tions providing a much more complex picture than in case of a standard chaotic system with only one unstable direction. Moreover, investigations of real systems are usually based on single scalar time series measurements. When such a time series is used to infer about higher dimensional dynamics, we can have some problems with the embedding procedure. For example, one should cope with a spurious folding appearing in projection from higher dimensional space to onedimensional subspace, as discussed, e.g., in Ref. [12].

\section{Conclusions}

We have shown that the generalized four-dimensional Lorenz system for convection in a horizontally magnetized viscous fluid layer exhibits quite intriguing features. It is rather surprising that this simple model can be derived from the full set of partial differential equations. Despite this, the complex behavior of the solutions results from nonlinearity and not from complicated laws. More specifically, depending on two control parameters of the model, by increasing the temperature difference and the magnetic field strength initially applied along the layer, one can switch-on and - off between nonperiodic chaotic, periodic (limit cycle), and equilibrium (fixed point) asymptotic solutions. In addition, because of fine structure illustrated in the space of both control parameters, the influence of the induced magnetic field on the properties of the fluid could be much more intricate than a simple stabilizing effect predicted by simplified analysis of influence of the magnetic field on convective motion discussed in standard textbooks (see, e.g., Ref. [6]). This is confirmed by identification of some physical situations where a weak field may have strong destabilizing effect on the fluid [2].

It is important to note that besides of the chaotic behavior well known for the Lorenz model with unmagnetized fluid we have also identified in the generalized Lorenz system a hyperchaotic dynamics, with two positive Lyapunov exponents appearing for some specific intervals of the values of the temperature gradient for some intensity of the applied magnetic field [17]. Therefore, in this paper, we have obtained new strange attractors, also in a hyperchaotic regime. Admittedly, this new type of chaos is only possible in at least fourdimensional systems; hence this results here from coupling between the anisotropic tension of magnetic field lines and magnetic viscosity. 
In fact, our analysis is able to grasp characteristic signatures of the hydromagnetic convection that can be relevant for observational identification of complex dynamical behavior, resulting in some intermittent energy bursts predicted by the generalized Lorenz model. Therefore, this new hyperchaotic analytical low-dimensional system characterized by both types I and III of intermittent energy release may provide an explanation of irregular convective dynamical processes, observed often in various plasmas in laboratory (fusion devices) and space, which will hopefully be analyzed in the future studies.

There is a general agreement that the value of the magnetic Prandtl number for plasma can range from very small $\left(\sigma_{\mathrm{m}} \ll 1\right)$, for terrestrial turbulent flows (geodynamo), liquid metals (laboratory dynamos), and stars $\left(\sim 10^{-7}\right)$, to rather high both for astrophysical objects (galaxies and clusters, $\sim 10^{29}$ ) and fusion devices. Although a direct assessment of such parameter is difficult in many situations, it would be useful to examine such cases using our model as some examples shown in Fig. 2, before going into numerical modeling magnetoconvection. Following these results one can expect that the actual behavior can be extremely complicated for the highest magnetic Prandtl numbers in laboratory and space plasmas.

Therefore, the obtained results could be important for explaining the processes leading to appearance of irregular nonperiodic dynamics in nature. In addition, in future it would be useful to perform numerical MHD simulations to recover some special cases described by the analytical generalized Lorenz model. Admittedly, it is plausible that still in order to get information about some phenomena described by the model from direct numerical simulations, one would require long time of instantaneous computations using available computational resources. Because the generalized Lorenz system exhibits complex behavior resulting from nonlinearity, it certainly provides a novel contribution to chaos theory that could hopefully still be of interest to the dynamical systems community.

Acknowledgements This work was supported by the National Science Center, Poland (NCN), through Grant No. 2014/15/B/ ST9/04782. The author thanks Marek Strumik for preparation of the figures. The absence of conflict of interest is certified.

Open Access This article is distributed under the terms of the Creative Commons Attribution 4.0 International License (http:// creativecommons.org/licenses/by/4.0/), which permits unre- stricted use, distribution, and reproduction in any medium, provided you give appropriate credit to the original author(s) and the source, provide a link to the Creative Commons license, and indicate if changes were made.

\section{References}

1. Antar, G.Y., Counsell, G., Yu, Y., Labombard, B., Devynck, P.: Universality of intermittent convective transport in the scrape-off layer of magnetically confined devices. Phys. Plasmas 10, 419-428 (2003). https://doi.org/10.1063/1. 1536166

2. Bajer, K., Mizerski, K.: Elliptical flow instability in a conducting fluid triggered by an external magnetic field. Phys. Rev. Lett. 110(10), 104503 (2013). https://doi.org/10.1103/ PhysRevLett.110.104503

3. Bergé, P., Pomeau, Y., Vidal, C.: Order within Chaos. Towards a Deterministic Approach to Turbulence. Wiley, New York (1984)

4. Boussinesq, J.: Theorie Analytique de la Chaleur. GauthierVillars, Paris (1903)

5. Busse, F.H.: Homogeneous dynamos in planetary cores and in the laboratory. Annu. Rev. Fluid Mech. 32, 383-408 (2000). https://doi.org/10.1146/annurev.fluid.32.1.383

6. Cowling, T.G.: Magnetohydrodynamics. Adam Hilger, Bristol (1976)

7. Dresvin, S.V., Amouroux, J.: Heat and mass transfer in plasma jets. Adv. Heat Transf. 40, 451-521 (2007). https:// doi.org/10.1016/S0065-2717(07)40005-3

8. Eckmann, J.-P., Ruelle, D.: Ergodic theory of chaos and strange attractors. Rev. Mod. Phys. 57, 617-656 (1985). https://doi.org/10.1103/RevModPhys.57.617

9. Itoh, K., Itoh, S.-I., Fukuyama, A., Yagi, M., Yazumi, M.: Prandtl number of toroidal plasmas. J. Phys. Soc. Jpn. 62(12), 4269-4276 (1993). https://doi.org/10.1143/JPSJ. 62.4269

10. Karpen, J.T., Antiochos, S.K., Dahlburg, R.B., Spicer, D.S.: The Kelvin-Helmholtz instability in photospheric flows. Effects of coronal heating and structure. Astrophys. J. 403, 769-779 (1993). https://doi.org/10.1086/172248

11. Landau, L.D., Lifshitz, E.M., Pitaevskii, L.P.: Electrodynamics of Continuous Media, vol. 8. Pergamon Press, Oxford (1984)

12. Letellier, C., Rossler, O.E.: Hyperchaos. Scholarpedia 2(7), 1936 (2007). https://doi.org/10.4249/scholarpedia.1936

13. Li, T.Y., Yorke, J.A.: Period three implies chaos. Am. Math. Mon. 82, 985-992 (1975). https://doi.org/10.1080/ 00029890.1975 .11994008

14. Lorenz, E.N.: Deterministic nonperiodic flow. J. Atmos. Sci. 20, 130-141 (1963). https://doi.org/10.1175/ 1520-0469(1963)020<0130:DNF>2.0.CO;2

15. Macek, W.M.: Intermittency in the generalized Lorenz model. In: Skiadas, C. (ed.) Chaotic Modeling and Simulation. Chaos International Conferences. International Journal of Nonlinear Science, vol. 4, pp. 323328 (2015). http://www.cmsim.org/images/1Proceedings_ CHAOS2015_L-M-453-550.pdf

16. Macek, W.M., Strumik, M.: Model for hydromagnetic convection in a magnetized fluid. Phys. Rev. E 82(2), 027301 (2010). https://doi.org/10.1103/PhysRevE.82.027301 
17. Macek, W.M., Strumik, M.: Hydromagnetic intermittent convection in a magnetized viscous fluid. Phys. Rev. Lett. 112(7), 074502 (2014). https://doi.org/10.1103/ PhysRevLett.112.074502

18. Nordlund, Å., Stein, R.F., Asplund, M.: Solar surface convection. Living Rev. Solar Phys. 6, 2 (2009). https://doi.org/ 10.12942/lrsp-2009-2

19. Oberbeck, A.: Ueber die Wärmeleitung der Flüssigkeiten bei Berücksichtigung der Strömungen infolge von Temperaturdifferenzen. Ann. Phys. (Leipzig) 243, 271-292 (1879). https://doi.org/10.1002/andp.18792430606

20. Pomeau, Y., Manneville, P.: Intermittent transition to turbulence in dissipative dynamical systems. Commun. Math. Phys. 74, 189-197 (1980). https://doi.org/10.1007/ BF01197757

21. Rayleigh, L.: On convecting currents in a horizontal layer of fluid when the higher temperature is on the under side. Philos. Mag. 32, 529-546 (1916). https://doi.org/10.1080/ 14786441608635602

22. Rossler, O.E.: An equation for hyperchaos. Phys. Lett. A 71, 155-157 (1979). https://doi.org/10.1016/ 0375-9601(79)90150-6

23. Rowlands, G., Sprott, J.C.: Extraction of dynamical equations from chaotic data. Phys. D Nonlinear Phenom. 58, 251-259 (1992). https://doi.org/10.1016/ 0167-2789(92)90113-2
24. Saltzman, B.: Finite amplitude free convection as an initial value problem-I. J. Atmos. Sci. 19, 329-341 (1962). https:// doi.org/10.1175/1520-0469(1962)019<0329:FAFCAA>2. 0.CO;2

25. Sarkovskii, A.N.: Coexistence of cycles of a continuous map of the line into itself. Ukr. Mat. Z. 16, 61-71 (1964). (in Russian)

26. Schuster, H.G.: Deterministic Chaos. An Introduction. VCH Verlagsgesellschaft, Weinheim (1988)

27. Sparrow, C.: The Lorenz Equations: Bifurcations. Chaos and Strange Attractors. Springer-Verlag, Berlin (1982)

28. Strumik, M., Macek, W.M.: Influence of dynamical noise on time series generated by nonlinear maps. Phys. D Nonlinear Phenom. 237, 613-618 (2008). https://doi.org/10.1016/ j.physd.2007.10.002

29. Strumik, M., Macek, W.M., Redaelli, S.: Discriminating additive from dynamical noise for chaotic time series. Phys. Rev. E 72(3), 036219 (2005). https://doi.org/10.1103/ PhysRevE.72.036219

30. Takens, F.: Forced oscillations and bifurcations. Comm. Math. Inst. Rijksuniv. 2, 1-111 (1974). https://www. taylorfrancis.com/books/e/9781420034288/chapters/10.12 01\%2F9781420034288-1 